Aportaciones para la enseñanza de la química con orientación a la protección ecológica.

\title{
Sostenibilidad y laboratorios escolares
}

\author{
Jaime Carrascosa, ${ }^{1} M^{a}$ Teresa Climent, $^{2} M^{a}$ Consuelo Dominguez, ${ }^{3}$ Lorena Paya ${ }^{4}$
}

\begin{abstract}
Sustainability and school laboratories) In this work we present a study of how environmental sustainability deals with on secondary school centres. The analysis includes the current status in teaching chemistry at secondary level: laboratory teaching, classroom books and the point of view of chemistry teachers about the environmental hazard of some of the chemicals used along in these tasks. In order to derive conclusions we start proposing the following questions:
\end{abstract}

1. Which dangerous substances for the environment can be found in the secondary school laboratories and how frequently are they used?

2. What is done with the products after a chemical reaction?

3. Are the chemistry teachers aware of the dangerous substances used and stored in their laboratories?

4. Which hazardous substances for the environment are proposed in the secondary chemistry books to perform practical works? Is the given information about their impact on the environment enough?

The strategies to answer these questions and the results obtained are exposed in this paper.

\section{Introducción}

Actualmente no es ninguna exageración afirmar que estamos viviendo una situación de verdadera emergencia planetaria, marcada por toda una serie de graves problemas estrechamente relacionados entre

\footnotetext{
${ }^{1}$ Universitat de València. Profesor colaborador del Departamento de Didáctica de las Ciencias.

Correo electrónico: jaime.carrascosa@uv.es

2 Laboratorio de Química General. Universitat de València.

Correo electrónico: Teresa.Climent@uv.es

3 Instituto Enseñanza Secundaria: La Vall de Segó (Valencia).

Correo electrónico: xelodominguez@yahoo.es

4 Instituto Enseñanza Secundaria: La Pobla de Segur (Lleida). Correo electrónico: lorena_paya@hotmail.com

Recibido:28 de marzo de 2007; aceptado: 5 de junio de 2007.
}

sí, tales como: superpoblación, contaminación, agotamiento de recursos naturales, cambio climático, pérdida de biodiversidad, lluvia ácida y destrucción de la capa de ozono. Dichos problemas, lejos de disminuir, son cada vez más intensos y extienden su impacto sobre todo el planeta. Así, en cuanto al calentamiento global, se afirma que la gravedad es tal, que si no se pone freno antes de una decena de años, después será ya demasiado tarde (Gore, 2007). No es pues de extrañar que desde Naciones Unidas se haya hecho un llamamiento para que todos los educadores nos impliquemos más en la formación de unos futuros ciudadanos capaces de hacer frente a estos problemas, institucionalizando la llamada Década para la Educación por un Futuro Sostenible, para el periodo 2005-2014 (Educadores por la sostenibilidad, 2007).

La participación del profesorado en la iniciativa anterior supone, entre otras cosas, la incorporación funcional de la problemática sobre la situación del mundo en el currículo. Existen, no obstante algunas dificultades generales que conviene tener en cuenta. En efecto, un primer obstáculo es la falta de información, ya que dos años después de haber comenzado la Década... todavía son muchos los profesores que desconocen su existencia. Además, se dan toda una serie de ideas preconcebidas al respecto, como la de que incorporar esa problemática en nuestras clases supone un desvío que resta efectividad al aprendizaje de los contenidos conceptuales propios de la asignatura; también hay quienes piensan que se exagera y que la situación no es tan grave, o que es muy poco lo que se puede hacer desde los centros escolares. Por otra parte, entre quienes sí tratan de incorporar estos temas en su enseñanza, son más los que hacen hincapié en la enumeración de los problemas que los que se detienen en la consideración y puesta en práctica de posibles soluciones, generando así una sensación de frustración e impotencia (Carrascosa, Gil-Pérez y Vilches, 2006).

No podemos entrar aquí en la discusión de todas las dificultades anteriores pero sí señalar que en el caso de las materias de ciencias, son muchas las ocasiones que permiten incorporar de forma funcional estos problemas y las posibles soluciones al pro- 


\section{EDUCACIÓN QUIIMICA PARA UN FUTURO SOSTENIBLE}

ceso de enseñanza y, lo que es más importante, desarrollar comportamientos coherentes con la idea de sostenibilidad a la vez que una actitud más positiva hacia el aprendizaje de los contenidos más conceptuales. Dicha incorporación se debe llevar a cabo simultáneamente en todos aquellos aspectos fundamentales para el aprendizaje, desde la introducción de conceptos y resolución de problemas a las prácticas de laboratorio y la propia evaluación. En este trabajo en concreto nos hemos centrado en analizar la contribución a la sostenibilidad que puede hacerse desde los laboratorios escolares de química. Para ello hemos comenzado por realizar una primera aproximación analizando la situación actual en los laboratorios de algunos centros de enseñanza secundaria.

\section{¿Qué problemas nos planteamos?}

En un estudio sobre la atención a la situación del mundo en la educación científica (Edwards et al., 2004), se afirma textualmente que los resultados correspondientes a profesores en formación y en activo y a libros de texto apoyan la idea de que la atención a la situación del mundo constituye una dimensión prácticamente ausente en la educación científica. Este estudio se realizó analizando de forma general las referencias y reflexiones que tanto profesores asistentes a distintos cursos de formación, como libros de texto, hacen respecto a los graves problemas generales que afectan a nuestra supervivencia. Recientemente, la comisión de educación del grupo Ecologistas en Acción, realizó un estudio sobre el llamado currículo oculto de los libros de texto en general (véase en: http://www.ecologistasenaccion.org/curriculumoculto). Las conclusiones a las que llegaron las resumieron enunciando que los libros de texto suspenden en sostenibilidad: confunden de forma sistemática el bienestar con el crecimiento económico, alaban las posibilidades de la tecnología sin cuestionar los problemas derivados del escaso debate y control social sobre los usos de la misma, etc. Las conclusiones de estudios como los anteriores y el llamamiento realizado por Naciones Unidas, dejan bien patente la necesidad de que todos los docentes nos impliquemos mucho más de lo que solemos hacerlo en la educación para la sostenibilidad, es decir, para avanzar hacia un desarrollo que satisfaga las necesidades de las generaciones presentes sin comprometer la capacidad de las generaciones futuras para satisfacer sus propias necesidades (Vilches y Gil, 2003). Con este objetivo hemos comenzado un trabajo sobre las prácticas de química en los laboratorios escolares de secundaria españoles, planteándonos de entrada los siguientes problemas:

\section{¿Suspenden en sostenibilidad los laboratorios \\ y las prácticas escolares de química?}

¿Cómo convertir el laboratorio escolar y las prácticas de química que se realicen en ellos en elementos que contribuyan a la educación para la sostenibilidad?

Antes que nada, conviene tener en cuenta el interés de los problemas que acabamos de plantear. En efecto, podría parecer que al tratarse de pequeños laboratorios que afectan únicamente al ámbito escolar, se trata de una cuestión menor, pero lo cierto es que son más de 4,500 los centros de secundaria existentes en España, con muchos productos químicos almacenados en ellos, de los cuales un porcentaje apreciable son sustancias peligrosas o muy peligrosas para el medio ambiente. Cabe pensar pues, que el grado de conocimiento del profesorado acerca de la toxicidad de los productos existentes en los laboratorios escolares y la gestión que se haga de los mismos y de los residuos que se generan, sí que pueden tener un impacto real apreciable sobre el medio ambiente y las personas.

No obstante, el interés del problema planteado no se reduce sólo a la consideración de su mayor o menor impacto medioambiental. Es preciso también tener en cuenta que no se puede pretender educar en la sostenibilidad si desde la propia escuela no se da ejemplo con todo tipo de comportamientos y acciones concretas coherentes con esta idea. A las prácticas de laboratorio siempre se les ha atribuido, acertadamente, un gran potencial motivador para el aprendizaje de las ciencias. Nuestra hipótesis es que, adecuadamente diseñadas, también pueden tener un gran potencial para la educación de los alumnos en la sostenibilidad. Propuestas didácticas como la llamada "química verde" (Montagut et al., 2006) y la "química en microescala" (Arnaiz, 2005), van precisamente en esa dirección.

Cabe plantearse pues, en primer lugar, cuál es la situación real de los laboratorios escolares y de las prácticas de química que se realizan en los mismos. En nuestra opinión, el problema de la sostenibilidad apenas se tiene en cuenta en los laboratorios escolares de secundaria y en la mayoría de las prácticas de química propuestas. Dicha opinión se basa, tanto en nuestra experiencia como profesores de física y química en diversos centros de secundaria como en algunos datos obtenidos mediante evaluaciones externas, tal como la realizada por la Secretaría de 
Salud Laboral del sindicato español Comisiones Obreras (CCOO), en el que se puso de manifiesto la existencia de riesgo químico muy importante en más de un $75 \%$ de los centros de enseñanza visitados (Mancheño, 2006).

Para conocer mejor la situación pensamos que era necesario obtener algunos datos cuantitativos respecto a ciertas cuestiones concretas:

$1^{\text {a) }}$ ¿Qué sustancias peligrosas para el medio ambiente existen en los laboratorios escolares de secundaria españoles y con qué frecuencia se utilizan?

$\left.2^{\mathrm{a}}\right)$ ¿Qué se hace con los productos que se obtienen y con los residuos que se generan?

$3^{a}$ ) ¿En qué medida el profesorado de química está informado del grado de peligrosidad de los productos que se manejan o se tienen almacenados en los laboratorios?

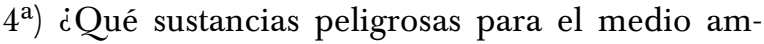
biente existen en las prácticas que se proponen en los libros de texto de secundaria y qué información se da sobre dicha peligrosidad?

Dar respuesta a las cuestiones anteriores constituye el objetivo fundamental de este trabajo.

Para investigar sobre las dos primeras cuestiones elaboramos una tabla inicial con una serie de productos químicos. Dicha tabla fue presentada a profesores de física y química de secundaria en activo, que tenían que indicar si en el laboratorio del centro disponían o no de cada uno de los productos en ella reseñados, así como la frecuencia con que lo utilizaban $(0=$ nunca, $1=$ muy poco, $2=$ poco, $3=$ normalmente, $4=$ frecuentemente). En cada caso debían señalar también qué hacían con los posibles residuos (si van a la papelera, se vierten por el fregadero, se recuperan, o qué otras acciones). Una vez rellenada la tabla, se les preguntaba además si disponían o no de un recinto separado del laboratorio para almacenar los residuos, si había alguna entidad que se ocupase de retirarlos del centro para su tratamiento y si tenían o no almacenadas algunas sustancias durante más de cinco años sin usarlas. Finalmente, se les invitaba a completar la lista de productos con otros que tuviesen, pero que no se hubieran contemplado en la tabla.

Para obtener datos con los que responder la tercera cuestión, utilizamos una segunda tabla que incluía los mismos productos que la primera (y algunos más que fueron incorporados posteriormente) y en la que se preguntaba qué grado de peligrosidad se consideraba que tenía cada uno de ellos $(1=$ nada o muy poco, $2=$ poco, $3=$ bastante, $4=$ muy peligroso, $5=$ no sé). En los productos calificados con un 3 o un 4 se solicitaba que indicasen, en su caso, algún otro menos peligroso que pudiera sustituirlo en alguna práctica de laboratorio concreta. Por último, completada la tabla, se pedía citar posibles acciones que pudieran llevarse a cabo en un laboratorio escolar para reducir el impacto ambiental y sobre la salud, tanto de las diversas sustancias utilizadas como de los residuos producidos.

En cuanto a la cuarta cuestión, para el análisis de los trabajos prácticos propuestos en los libros de texto de física y química utilizados habitualmente en la enseñanza secundaria, se elaboró una tercera tabla en la que para cada libro se recogieron los siguientes datos: total de prácticas de química propuestas; total de productos químicos diferentes implicados (excepto agua, oxígeno e indicadores); total de productos peligrosos o muy peligrosos para el medio ambiente y número de veces que aparece cada uno. También analizamos en cada producto calificado como peligroso o muy peligroso, si en el texto se incluía alguna información al respecto advirtiendo, al menos, de la necesidad de impedir su incorporación al medio ambiente.

Para establecer el grado de peligrosidad de cada producto utilizamos fundamentalmente las Fichas Internacionales de Seguridad Química elaboradas por iniciativa del Programa Internacional para la Seguridad Química en el que colaboran las Naciones Unidas, la Organización Internacional del Trabajo y la Organización Mundial de la Salud. Uno de los objetivos de dicho programa es llevar a cabo evaluaciones de los riesgos ocasionados por las distintas sustancias químicas en la salud y en el medio ambiente (véase: http://www.mtas.es/insht/ipcsnspn/ nspnsyn.htm y también http://www.fichasdeseguridad.com). En el Anexo que se da al final de este artículo se incluye una selección de productos considerados como peligrosos o muy peligrosos para el medio ambiente, indicando el nombre de cada uno y una breve descripción de sus efectos. Al elaborar dicha selección hemos intentado que la mayoría de los productos peligrosos que suelen estar presentes en los laboratorios escolares o que se proponen para utilizar en las prácticas que se incluyen en los libros de texto se encuentren en ella aunque, por supuesto, se trata de una relación en constante evolución conforme vayan incorporándose nuevos productos. 


\section{Productos químicos peligrosos en los} laboratorios escolares. ¿Cuál es la situación?

Los resultados que se presentan en la tabla I, corresponden a un colectivo de 20 docentes de física y química en activo que trabajan en otros tantos centros escolares de secundaria. Los datos fueron obtenidos en el curso 2006-07 y los consideramos como una primera aproximación sobre la que convendría profundizar en un futuro ampliando la muestra considerada.

En la primera columna figuran los productos peligrosos detectados inicialmente, resaltando en negrita los considerados, de acuerdo con la bibliografía consultada, como muy peligrosos para el medio ambiente; en la segunda columna se informa acerca de la disponibilidad de cada uno, dando el porcentaje de centros que lo tienen en sus laboratorios; en la tercera se indica una estimación de la frecuencia con que se usa cada producto en una escala que va del 0 (nunca) al 4 (frecuentemente); finalmente, en la cuarta columna se indica el porcentaje de centros en los que los restos se eliminan por el fregadero o tirándolos a la papelera.

El análisis de los datos de la tabla I, nos lleva a realizar las siguientes consideraciones:

En la mayoría de los centros se pueden encontrar productos considerados muy tóxicos o peligrosos, como el ácido fluorhídrico (en el 75\% de los centros), mercurio y algunos derivados (en más del 80\% de los centros), benceno (en el 56\%), sodio metálico (en el 75\%) y que, sin embargo, apenas se utilizan. Ninguno de los mencionados llega al 1 en cuanto a frecuencia media de uso.

Al mismo tiempo se puede apreciar que en la gran mayoría de centros hay otros productos también muy peligrosos para el medio ambiente y que se usan con mucha mayor frecuencia que los anteriores como, por ejemplo, el tetracloruro de carbono (presente en más del 85\% de centros y con una frecuencia media de uso de 1.3), el sulfato de cobre II (disponible en el 100\% de los centros consultados y con una frecuencia media de uso superior a 3), el dicromato de potasio (en el $87 \%$ de centros y frecuencia media de uso de 2), o el permanganato de potasio (presente en el 100\% de los centros y frecuencia media de uso de 2.1).

$\mathrm{Al}$ analizar los datos de la última columna, constatamos que el destino más común de los residuos es el fregadero o la papelera.

Otros resultados a tener en cuenta son:

En más del 90\% de los centros consultados no
Tabla I. Algunos productos presentes en los laboratorios escolares. Profesores de Física y Química de Centros Públicos de Secundaria. N=20. Valencia. 2006.

Frecuencia de uso: $0=$ nunca; $1=$ muy poco; $2=$ poco; $3=$ normalmente; $4=$ frecuentemente.

\begin{tabular}{|c|c|c|c|}
\hline Producto & $\begin{array}{c}\text { Disponible } \\
\%\end{array}$ & $\begin{array}{c}\text { Frecuencia } \\
\text { media de uso }\end{array}$ & $\begin{array}{l}\text { Papelera o } \\
\text { Fregadero }\end{array}$ \\
\hline Ácido fluorhídrico & 75 & 0.1 & 100 \\
\hline Ácido sulfúrico & 100 & 2.7 & 75 \\
\hline Ácido tartárico & 62.5 & 0.7 & 100 \\
\hline Amonio nitrato & 75 & 1.3 & 71.3 \\
\hline Bario cloruro & 81.3 & 1.3 & 77.8 \\
\hline Benceno & 56.3 & 0.9 & 100 \\
\hline Carbono tetracloruro & 87.5 & 1.3 & 60 \\
\hline Cobalto (II) cloruro & 37.5 & 1.5 & 60 \\
\hline Cobre (II) sulfato & 100 & 3.1 & 62.5 \\
\hline Cobre (II) cloruro & 62.5 & 1.3 & 28.6 \\
\hline Manganeso (IV) óxido & 87.5 & 1.1 & 71.5 \\
\hline Mercurio & 87.5 & 0.8 & 33.3 \\
\hline Mercurio (II) cloruro & 81.3 & 0.2 & 100 \\
\hline Mercurio (I) nitrato & 81.3 & 0.3 & 50 \\
\hline Mercurio (II) nitrato & 62.5 & 0.3 & 50 \\
\hline Mercurio (II) óxido & 81.3 & 0.6 & 50 \\
\hline Naftaleno & 43.8 & 1.3 & 50 \\
\hline Plata nitrato & 100 & 2.3 & 73.3 \\
\hline Plomo & 87.5 & 1.1 & 33.3 \\
\hline Plomo (II) carbonato & 43.8 & 0.1 & - \\
\hline Plomo (IV) óxido & 18.8 & 0 & - \\
\hline Potasio dicromato & 87.5 & 2.0 & 63.7 \\
\hline Potasio permanganato & 100 & 2.1 & 53.8 \\
\hline Sodio & 75 & 0.8 & 100 \\
\hline Sodio hidróxido & 93.8 & 2.9 & 80 \\
\hline Sodio tiosulfato & 68.8 & 0.7 & 100 \\
\hline Xileno & 43.8 & 1.2 & 100 \\
\hline Yodo & 93.8 & 2.3 & 71.5 \\
\hline Zinc cloruro* & $68.8^{*}$ & $0.5^{*}$ & $27.3^{*}$ \\
\hline Zinc sulfato & 68.8 & 1.5 & 42.3 \\
\hline Aceite & 75 & 1.9 & 27.3 \\
\hline
\end{tabular}

* En el caso del cloruro de zinc, tenemos constancia de que éste se produce en muchas ocasiones como consecuencia de la reacción entre ácido clorhídrico y zinc, bien sea para obtener hidrógeno o para otros usos como la determinación de la fórmula empírica del compuesto o el estudio de factores que afectan a la velocidad de reacción. Por tanto, la existencia y frecuencia de uso son, en realidad, más altas que las reseñadas. Análogas consideraciones pueden hacerse respecto de otros productos como, por ejemplo, el 1,2-dibromoetano, una sustancia considerada como muy tóxica para el medio ambiente (especialmente el acuático) que aunque no se disponga de ella en el laboratorio, se suele producir al investigar la existencia de insaturaciones utilizando bromo y eteno; o el yoduro de plomo (II) que precipita en forma de pequeños cristalitos amarillos en la conocida reacción entre yoduro de potasio y nitrato de plomo (II) ambos en disolución acuosa. 
se dispone de un recinto separado en el que almacenar los restos que se generan en las prácticas y en ninguno de ellos se da ninguna actuación que consista en la recogida periódica de productos peligrosos que se hayan generado o que hayan caducado, por alguna empresa especializada en el tratamiento de los mismos para evitar o disminuir su impacto ambiental.

Por otra parte, en el 70\% de los centros encuestados constatamos que se dispone de sustancias que no han sido utilizadas desde hace más de cinco años. Éstas suelen estar almacenadas dentro de los laboratorios en armarios situados en el mismo lugar donde los alumnos realizan las prácticas y en ocasiones se dan clases, con el consiguiente riesgo de intoxicación ante botellas mal cerradas o con cierres deteriorados. Baste pensar, por ejemplo, en la mayoría de disol-

Tabla II. Resultados de la encuesta sobre el grado de percepción de peligrosidad atribuido a algunos productos.

PF: 24 (Licenciados en Química o Farmacia: 21, Física: 3). Universidad de

Valencia. Curso: 06/07.

PA: 18 (Profesores de Física y Química de secundaria en activo).

Valencia. Curso: 06/07

Peligrosidad: $\mathbf{1}=$ Nada o muy poco; $\mathbf{2}$ = Un poco; $\mathbf{3}=$ Bastante;

$4=$ Muy peligroso

\begin{tabular}{l|c|c|c|c|c|c}
\multicolumn{1}{c|}{ PRODUCTO } & \multicolumn{2}{c|}{ Peligrosidad } & \multicolumn{2}{c|}{$\%$ No lo sé } & \multicolumn{2}{c}{$\begin{array}{c}\text { Muy } \\
\text { peligroso }\end{array}$} \\
\hline & PF & PA & PF & PA & PF & PA \\
\hline Ácido fluorhídrico & 3.7 & 3.6 & 11.1 & 5.6 & 61.1 & 72.2 \\
\hline Ácido sulfúrico & 4.0 & 3.4 & 0.0 & 0.0 & 100 & 50.0 \\
\hline Amonio nitrato & 2.7 & 2.3 & 44.4 & 21.7 & 11.1 & 5.6 \\
\hline Benceno & 3.4 & 3.6 & 16.7 & 5.6 & 44.4 & 61.1 \\
\hline Carbono tetracloruro & 3.1 & 3.6 & 33.3 & 0.0 & 33.3 & 66.7 \\
\hline Cobre (II) sulfato & 2.8 & 2.2 & 55.6 & 5.7 & 5.6 & 5.6 \\
\hline Manganeso (IV) óxido & 1.9 & 2.6 & 55.6 & 16.7 & 0.0 & 5.6 \\
\hline Mercurio & 3.6 & 3.7 & 0.0 & 0.0 & 61.1 & 72.2 \\
\hline Sales de mercurio & 3.8 & 3.5 & 27.8 & 11.1 & 55.6 & 66.7 \\
\hline Naftaleno & 2.3 & 2.7 & 27.8 & 22.2 & 16.7 & 11.1 \\
\hline Plata nitrato & 2.3 & 2.3 & 5.6 & 5.6 & 11.1 & 5.6 \\
\hline Plomo & 3.1 & 2.7 & 5.6 & 0.0 & 55.6 & 27.8 \\
\hline Plomo (II) carbonato & 3.3 & 3.2 & 27.8 & 5.6 & 38.9 & 44.4 \\
\hline Potasio dicromato & 2.4 & 2.9 & 61.1 & 5.6 & 5.6 & 22.2 \\
\hline Potasio & 2.4 & 3.8 & 38.9 & 11.1 & 11.1 & 5.6 \\
\hline permanganato & & & & & & \\
\hline Sodio hidróxido & 2.1 & 2.9 & 22.2 & 0.0 & 38.9 & 16.7 \\
\hline Sodio tiosulfato & 2.9 & 2.1 & 50.0 & 27.8 & 11.1 & 5.6 \\
\hline Yodo & 2.2 & 2.2 & 16.7 & 0.0 & 11.1 & 11.1 \\
\hline Zinc cloruro & 1.8 & 2.3 & 44.4 & 11.1 & 11.1 & 5.6 \\
\hline Aceite & 1.1 & 1.9 & 0.0 & 0.0 & 0.0 & 11.1 \\
\hline & & & & & & \\
\hline
\end{tabular}

ventes orgánicos o en otras sustancias como el ácido fluorhídrico, muy tóxicas para el ser humano y que por evaporación a temperatura ambiente se pueden alcanzar rápidamente concentraciones nocivas. En algunos laboratorios de centros escolares antiguos, además de los ya reseñados, se pueden encontrar otros productos muy peligrosos como acetato de uranio, dicromato de amonio, cianuro de potasio, cianuro de sodio, fenol, etc., que pueden llevar almacenados más de 15 años, haciendo de dichos laboratorios verdaderas bombas de relojería.

A continuación (a título de ejemplo), se reproducen algunos comentarios relevantes, realizados por los profesores encuestados, relacionados con el tratamiento de residuos:

- Los sólidos se tiran a la papelera y los líquidos se vierten a la pila con el grifo abierto.

- Recuperamos el $\mathrm{CuSO}_{4}$ cristalizado pero sólo una parte.

- Recuperamos el $\mathrm{PbI}_{2}$ pero siempre nos queda una disolución acuosa con los restos de la reacción entre el $\mathrm{Pb}\left(\mathrm{NO}_{3}\right)_{2}$ y el $\mathrm{KI}$, que eliminamos por el fregadero.

- Para eliminar bases y ácidos fuertes suelo neutralizarlos antes de verterlos a la pila y lo mismo hago con las bases fuertes.

\section{Conocimiento sobre la peligrosidad para el medio ambiente de los productos existentes en los laboratorios escolares}

Los resultados que se presentan en la tabla II corresponden a dos colectivos: uno formado por 18 profesores de física y química de secundaria en activo, diferentes a los implicados en la tabla I (encabezados como PA) y el otro por 24 licenciados universitarios de ciencias que en 2006-07 realizaron el Curso de Aptitud Pedagógica, que podemos considerar como "profesores en formación" (encabezados como PF).

Ambos colectivos fueron consultados acerca del grado de peligrosidad de una lista de productos habituales en los laboratorios escolares. En la tabla II se dan los datos obtenidos relativos a algunos de los productos que integraban dicha lista (se seleccionaron los peligrosos o muy peligrosos para el medio ambiente y se muestran en la primera columna). En la segunda columna se recogen los resultados en cuanto a la peligrosidad atribuida a cada uno mediante una nota que varía entre 1 (nada o muy poco) y 4 (muy peligroso); en la tercera se indica el porcentaje de profesores que manifiesta desconocer el grado de peligrosidad y en la cuarta el porcentaje 
que califica con una nota de 4 (muy peligroso) al producto en cuestión. Para facilitar el análisis hemos procedido a resaltar en negrita los productos muy peligrosos para el medio ambiente.

Los datos obtenidos parecen indicar que los dos colectivos consultados son conscientes de la peligrosidad para el medio ambiente y las personas de muchos productos como: el ácido fluorhídrico, benceno, tetracloruro de carbono, mercurio y sus derivados, plomo y sus derivados, a los que califican en promedio como bastante peligrosos. Sin embargo, no ocurre lo mismo en otros casos como, por ejemplo, el sulfato de cobre (II), cloruro de zinc, yodo, o aceite, que son valorados como muy poco o poco peligrosos. Particular atención merecen productos como el cloruro de zinc, el aceite, el permanganato de potasio, el dicromato de potasio o el yodo, que son calificados como muy peligrosos por un porcentaje muy escaso de profesores tanto en formación como en activo (en general, menos del 12\%) lo que contrasta con su elevada peligrosidad real. Así, por ejemplo, las sales de cromo VI, son compuestos carcinógenos demostrados y muy tóxicos para el medio acuático, de efectos prolongados en la cadena trófica.

En cuanto a posibles acciones a llevar a cabo para minimizar el impacto ambiental y sobre las personas, las propuestas que más se repiten entre quienes contestan a esta cuestión son: disponer de recipientes separados donde almacenar residuos, diluir o neutralizar antes de verter a la pila, tener en cuenta las incompatibilidades de almacenamiento y también otras relacionadas con la prevención de accidentes directos, como llevar bata y gafas de protección. Sin embargo, no se menciona en ningún caso la opción de emplear cantidades de productos muy pequeñas en la realización de las prácticas.

Finalmente, respecto a la posibilidad de utilizar productos menos peligrosos en determinadas prácticas, no se hacen apenas propuestas concretas. Ocasionalmente se menciona la utilización de tolueno o xileno en lugar de benceno y la conveniencia de eliminar definitivamente de los laboratorios escolares algunas sustancias como el ácido fluorhídrico, el benceno o el sodio metal.

\section{Productos químicos peligrosos en los libros de texto. ¿Cuál es la situación?}

El libro de texto constituye un material muy importante para la enseñanza y aprendizaje de las ciencias, hasta el punto de que las posibles innovaciones y reformas que se pretendan llevar a cabo en la educación se hallan condicionadas, en gran medida, por el grado en que las mismas se vean reflejadas en los libros de texto que habitualmente se utilizan. De acuerdo con ello, en el caso de la sostenibilidad será fundamental la atención que se presta a esta problemática en las prácticas de laboratorio incluidas en los libros de texto.

En este trabajo hemos analizado un total de 39 libros de texto de enseñanza secundaria, todos ellos con prácticas de química, de los cuales 11 han sido de $3^{\circ}$ de Enseñanza Secundaria Obligatoria (ESO) para alumnos de entre 14 y 15 años, otros 11 de $4^{\circ}$ de ESO (15-16 años), ocho textos de $1^{\circ}$ de Bachillerato (16-17 años) y nueve de $2^{\circ}$ de Bachillerato (17-18 años). Los resultados obtenidos se exponen en la tabla III en la que en la primera columna se enumeran los productos peligrosos o muy peligrosos para el medio ambiente que aparecen en las prácticas incluidas en los libros de texto de secundaria analizados y en las cuatro columnas siguientes el número de prácticas en las que se propone utilizar cada uno de dichos productos. En el encabezamiento de cada columna se especifica el nivel educativo y, entre paréntesis, el número total de prácticas de química propuestas en dicho nivel. Se han resaltado en negrita los considerados como muy peligrosos para el medio ambiente de acuerdo con la bibliografía consultada al respecto (véase el anexo al final). En la sexta columna se da el total de prácticas de química de secundaria (siempre refiriéndonos a los 39 libros analizados) en las que se propone cada producto $y$, por último, en la séptima (encabezada por MA), se especifica el total de advertencias realizadas o de informaciones que tengan que ver con la peligrosidad del producto para el medio ambiente y la consiguiente necesidad de no eliminarlo por el desagüe ni echándolo a la papelera.

De acuerdo con los datos de la tabla III, vemos que el total de prácticas de química analizadas asciende a 325. En ellas se propone el uso de 51 productos diferentes peligrosos para el medio ambiente (de los cuales, al menos 15 se consideran como muy peligrosos). Si ignoramos el hecho de que un mismo producto pueda ser propuesto en distintas prácticas, obtenemos un total de 354 productos peligrosos, lo que supone una media de algo más de uno de dichos productos por cada práctica propuesta. Si nos limitamos a los considerados como muy peligrosos esta cantidad se reduce a 0.37 o lo que es equivalente: 
En promedio, en una de cada tres prácticas que se incluyen en los textos de secundaria analizados, se propone la utilización de un producto muy peligroso para el medio ambiente.
No obstante, lo verdaderamente preocupante es que sólo en una de las 325 prácticas analizadas se advierte de la peligrosidad para el medio ambiente de un producto (el dicromato de potasio). Las muy escasas advertencias en cuanto a la peligrosidad de

Tabla III. Productos peligrosos o muy peligrosos en libros de texto de secundaria. 325 prácticas.

\begin{tabular}{|c|c|c|c|c|c|c|}
\hline PRODUCTOS & $3^{\circ}$ ESO $(81$ & $4^{\circ}$ ESO (54) & $1^{\circ}$ BACH (52) & $2^{\circ} \mathrm{BACH}(138)$ & $\mathbf{N}$ & MA \\
\hline Aceite & 5 & 2 & 4 & 1 & 12 & 0 \\
\hline Ácido fosfórico & - & - & - & 1 & 1 & 0 \\
\hline Ácido sulfúrico & 7 & 4 & 3 & 14 & 28 & 0 \\
\hline Ácido tartárico & 1 & - & - & - & 1 & 0 \\
\hline Aluminio sulfato & - & - & - & 2 & 2 & 0 \\
\hline Amonio dicromato & - & - & - & 1 & 1 & $\mathbf{0}$ \\
\hline Amonio nitrato & - & - & - & 4 & 4 & 0 \\
\hline Bario cloruro & 1 & - & 2 & 2 & 5 & 0 \\
\hline Bario hidróxido & 1 & - & - & 2 & 3 & 0 \\
\hline Bario nitrato & - & - & - & 1 & 1 & 0 \\
\hline Benceno & - & 2 & 1 & 2 & 5 & 0 \\
\hline Benzaldehído & - & - & 1 & - & 1 & 0 \\
\hline Bromo & 1 & - & 1 & 2 & 4 & 0 \\
\hline Carbono tetracloruro & 2 & - & 4 & 4 & 10 & 0 \\
\hline Cloro & 2 & 4 & - & - & 6 & 0 \\
\hline Cloroformo & - & - & - & 1 & 1 & 0 \\
\hline Cobalto (II) cloruro & - & - & - & 3 & 3 & 0 \\
\hline Cobre (II) cloruro & 4 & 1 & - & 1 & 6 & 0 \\
\hline Cobre (II) nitrato & 1 & 3 & - & - & 4 & 0 \\
\hline Cobre (II) sulfato & 10 & 4 & 4 & 8 & 26 & 0 \\
\hline Diclorometano & - & - & - & 1 & 1 & 0 \\
\hline Estroncio nitrato & - & - & - & 1 & 1 & 0 \\
\hline Fenol & - & - & - & 2 & 2 & 0 \\
\hline Formaldehído & - & - & - & 1 & 1 & 0 \\
\hline Gasolina & - & - & 3 & - & 3 & 0 \\
\hline Hierro (III) nitrato & 1 & - & - & - & 1 & 0 \\
\hline Magnesio & 6 & 2 & 1 & 5 & 14 & 0 \\
\hline Manganeso (IV) óxido & 2 & 3 & 2 & 4 & 11 & 0 \\
\hline Mercurio & - & - & - & 3 & 3 & 0 \\
\hline Naftaleno & 3 & 3 & 3 & 4 & 13 & 0 \\
\hline Plata nitrato & 6 & 1 & 5 & 4 & 16 & 0 \\
\hline Plomo & - & 2 & - & 1 & 3 & 0 \\
\hline Plomo (II) yoduro & & 1 & 1 & - & 2 & 0 \\
\hline Plomo (II) nitrato & 1 & 1 & 7 & 1 & 10 & 0 \\
\hline Plomo (IV) óxido & - & 2 & & 1 & 3 & 0 \\
\hline Plomo acetato & - & - & - & 1 & 1 & 0 \\
\hline Potasio & - & 1 & - & 1 & 2 & 0 \\
\hline Potasio clorato & 2 & 2 & - & 1 & 5 & 0 \\
\hline
\end{tabular}


Tabla III (continúa)...

\begin{tabular}{l|c|c|c|c|c|c}
\hline \multicolumn{1}{c|}{ PRODUCTOS } & $\mathbf{3}^{\mathbf{0}}$ ESO (81 & $\mathbf{4}^{\mathbf{0}}$ ESO (54) & $\left.\mathbf{1}^{\mathbf{0}} \mathbf{B A C H} \mathbf{5 2}\right)$ & $\mathbf{2}^{\mathbf{0}}$ BACH (138) & $\mathbf{N}$ & MA \\
\hline Potasio dicromato & - & 2 & 1 & 3 & 6 & 1 \\
\hline Potasio hidróxido & 1 & 1 & 1 & - & 3 & 0 \\
\hline Potasio nitrato & 1 & 2 & 1 & 1 & 5 & 0 \\
\hline Potasio permanganato & 2 & 1 & 2 & 4 & $\mathbf{9}$ & 0 \\
\hline Sodio & - & 1 & 1 & 2 & 4 & 0 \\
\hline Sodio bisulfito & - & - & - & 3 & 3 & 0 \\
\hline Sodio hidróxido & 12 & 11 & 8 & 36 & 67 & 0 \\
\hline Sodio nitrato & 2 & 1 & & 1 & 4 & 0 \\
\hline Sodio tiosulfato & 1 & 2 & & & 3 & 0 \\
\hline Xileno & - & - & - & 1 & 1 & 0 \\
\hline Yodo & 6 & 1 & 5 & 8 & $\mathbf{2 0}$ & 0 \\
\hline Zinc cloruro & 3 & 3 & 6 & - & $\mathbf{1 2}$ & 0 \\
\hline Zinc sulfato & - & - & - & 1 & 1 & 0 \\
\hline
\end{tabular}

los productos manejados se limitan a llamadas de atención sobre la necesidad de que una determinada práctica se realice con la supervisión del profesor o sobre el posible peligro físico que para los alumnos supone alguna actividad concreta como, por ejemplo, el manejo de disoluciones concentradas de ácidos o bases fuertes, o el desprendimiento de gases tóxicos tales como óxidos de nitrógeno o cloro.

Otros datos de interés son:

Los diferentes productos peligrosos o muy peligrosos para el medio que más se proponen son un total de 13 (en negrita los considerados como muy peligrosos para el medio ambiente):

Permanganato de potasio (9 prácticas), nitrato de plomo (10 prácticas), tetracloruro de carbono (10 prácticas), óxido de manganeso IV (11 prácticas), cloruro de zinc (12 prácticas), aceite (12 prácticas), naftaleno (13 prácticas), nitrato de plata (16 prácticas), magnesio (14 prácticas), yodo (20 prácticas), sulfato de cobre II (26 prácticas), ácido sulfúrico (28 prácticas), hidróxido de sodio (67 prácticas).

Si comparamos los resultados anteriores con los de la tabla I, nos podemos dar cuenta de la coherencia existente entre ambos, ya que los profesores de química de enseñanza secundaria utilizan la mayoría de estos productos con una frecuencia bastante alta (por ejemplo, 3.1 para el sulfato de cobre, 2.7 para el ácido sulfúrico, 2.9 para el hidróxido de sodio, 2.1 para el permanganato de potasio o 2.3 para el nitrato de plata). En el caso del cloruro de zinc (y otros similares) ya hemos comentado que al tratarse de sustancias que se obtienen también como productos finales de determinadas reacciones químicas, la fre- cuencia real es más alta que la que aparece en la tabla.

\section{Conclusiones finales, recomendaciones y perspectivas}

Los datos de este trabajo se han obtenido de los laboratorios de 20 centros públicos de enseñanza secundaria, 18 profesores de física y química en activo, 24 profesores en formación y 325 prácticas de química de un total de 39 libros de texto de secundaria españoles.

A falta de otros trabajos posteriores utilizando muestras más amplias, los resultados obtenidos parecen indicar que tanto en los laboratorios escolares de secundaria como en las prácticas de química propuestas en los libros de texto, se precisa una mayor atención a la sostenibilidad.

Una conclusión como la anterior se basa en la constatación de que:

En la mayoría de los laboratorios escolares analizados se acumulan diversas sustancias muy peligrosas para el medio ambiente, tales como benceno, ácido fluorhídrico, mercurio y compuestos de mercurio, algunos compuestos de plomo, etc. Todos esos productos han venido siendo suministrados por la administración educativa a los centros públicos de enseñanza cuando comienzan a ejercer su actividad.

Una gran parte del profesorado de química en formación y en activo conoce la peligrosidad para el medio ambiente de muchos productos químicos como benceno, mercurio, ácido sulfúrico, plomo, etc., pero esos mismos profesores desconocen que algunos productos, que suelen manejar frecuente- 
mente, como el sulfato de cobre (II), el cloruro de zinc o el yodo, también son muy peligrosos para el medio ambiente.

$\mathrm{Al}$ menos en los centros consultados por nosotros, no existe ningún protocolo establecido por el departamento de física y química sobre qué hacer con los residuos producidos en el laboratorio ni con los productos no deseados u obsoletos en donde se plantee seriamente una gestión respetuosa con el medio ambiente, siendo el destino más habitual de los residuos producidos en las prácticas, el fregadero y la papelera.

La situación descrita es coherente con la que se da en los libros de texto editados por las principales editoriales españolas. Recordemos, por ejemplo, que en una de cada tres prácticas de química de secundaria se propone la utilización de un producto considerado como muy peligroso para el medio ambiente sin que se haga ninguna advertencia al respecto, ni se incluyan pautas a seguir para la adecuada eliminación de los residuos generados, mostrando que esta problemática no ha sido, todavía, tenida en cuenta con la seriedad que merece.

De acuerdo con los resultados anteriores consideramos fundamental incorporar la educación para la sostenibilidad como una parte esencial de la formación inicial y permanente del profesorado de ciencias de secundaria y que dentro de ella se preste la atención necesaria a las prácticas de laboratorio y a todo lo relacionado con ellas, desde la manipulación de los productos a la gestión de los residuos. Esto es algo que se viene haciendo ya en algunos ámbitos universitarios (ver, por ejemplo: http:// www.uv.es/DSSQA/servicio/medio_ambiente), pero que está prácticamente ausente en la enseñanza secundaria.

Proponemos que las autoridades educativas doten del presupuesto adecuado para la revisión de todos los productos peligrosos y muy peligrosos que se acumulan en los laboratorios de secundaria. Retirada, a través de un gestor autorizado de residuos, de aquellos que no se utilizan así como los que se generan en la realización de las prácticas. Adecuación de las instalaciones para el almacenaje de los productos de laboratorio, de modo que queden separados del lugar de trabajo. Que se impulse por parte de la administración el proceso de compra de los reactivos según las necesidades, siendo el profesorado implicado en la realización de las prácticas el que decida qué y cuándo comprar. Revisión de los libros de química de secundaria, para que incluyan las advertencias sobre la peligrosidad para el medio ambiente de los reactivos químicos, contribuyendo de este modo a una educación para la sostenibilidad dentro del colectivo de profesores de secundaria y por tanto de sus estudiantes.

Una iniciativa de gran interés es la transformación de las prácticas de química que más frecuentemente se realizan en secundaria en prácticas de química a microescala, utilizando así cantidades mínimas de productos. Por otra parte, la educación ambiental ha de tener, para ser efectiva, un carácter global (Carrascosa, et al., 2006) y afectar a su vez a otros aspectos también esenciales para la enseñanza y aprendizaje de las ciencias como son los conceptos teóricos, los problemas o la propia evaluación. Todos ellos constituyen importantes líneas de investigación didáctica en las que los profesores deberíamos implicarnos para conseguir formar ciudadanos y ciudadanas capaces de tomar decisiones sobre cuestiones ambientales desarrollando su pensamiento crítico (Jiménez-Aleixandre, López-Rodríguez y Pereiro, 2006) y su capacidad para enfrentarse a los graves problemas que afectan a nuestro planeta y con él a toda la humanidad.

Como ya hemos señalado, los resultados que aquí presentamos son una primera aproximación de un estudio concebido a más a largo plazo, en el que nos proponemos, en primer lugar, ampliar el trabajo aquí expuesto. Por otra parte, queremos utilizar también todo el potencial que ofrecen las prácticas a microescala en cuanto a la educación para la sostenibilidad y a su capacidad para familiarizar con la metodología científica, realizando cursos de formación didáctica del profesorado de secundaria con ellas y analizando los resultados obtenidos.

\section{Referencias}

Arnaiz, F.J. Química en microescala en los laboratorios de Química inorgánica y organometálica. Educación Química, 16 (4), 504-509. 2005.

Carrascosa, J., Gil-Pérez, D. y Vilches, A. Concepciones espontáneas sobre la situación del mundo: un aspecto ignorado en la investigación sobre concepciones alternativas. Educación Cubana. La Habana, Cuba, 2006.

Carrascosa, J., Gil-Pérez, D., López-Alcantud, J. y Vilches, A. La educación para la sostenibilidad en el currículo de Física: El estudio de la energía como ejemplo privilegiado para abordar la situación del mundo. Educación Cubana. La Habana, Cuba, 2006.

Ecologistas en acción. Los libros de texto suspenden en 
sostenibilidad. Consultado por última vez en marzo 19. 2007, en la URL: http://www.ecologistas enaccion.org/curriculumoculto

Educadores por la sostenibilidad. Consultada por última vez en marzo 19, 2007, en la URL: http://www.oei.es/decada/compromiso.htm

Edwards, M., Gil-Pérez, D., Vilches, A. y Praia, J. La atención a la situación del mundo en la educación científica. Enseñanza de las ciencias, 22 (1), 47-63. 2004.

Fichas de datos de seguridad de sustancias químicas peligrosas. Consultado por última vez en marzo 19. 2007 en la URL: http://www.mtas.es/ insht/ipcsnspn/nspnsyn.htm

Fichas Internacionales de Seguridad Química. Consultado por última vez en marzo 19.2007, en la URL: http://www.mtas.es/insht/ipcsnspn/nspnsyn.htm

Gore, Al. Una verdad incómoda. La crisis planetaria del calentamiento global y cómo afrontarla. Gedisa. Barcelona. España. 2007

Jiménez-Aleixandre, M. P., López-Rodríguez, R. y Pereiro, M.C. La educación ambiental en el aula: pensamiento crítico y uso de conceptos científicos. Alambique, 48, 50-56. 2006.

Mancheño, C. Agentes químicos en la enseñanza. Trabajadores de la Enseñanza. 272, 18-19. 2006

Montagut, P., Nieto, E. y Sansón, C. Química verde y microescala: por un futuro mejor. Alambique, 47, 86-94. 2006.

Servei de seguretat, salut i qualitat ambiental de la Universitat de València. Consultado por última vez en marzo de 2007, en la URL: http:// www.uv.es/DSSQA/servicios/medio_ambiente

Vilches, A. y Gil-Pérez, D. Construyamos un futuro sostenible. Diálogos de supervivencia. Cambridge University Press, Madrid, España, 2003.

\section{Anexo. Algunos productos peligrosos para el medio ambiente}

1,2-Dibromoetano $\quad$ Muy tóxico, nocivo para el medio ambiente, especialmente el acuático. Posible carcinógeno.

\begin{tabular}{l|l}
\hline Aceite & Nocivo para el medio acuático. \\
\hline Acetaldehído & Nocivo para organismos acuáticos. \\
\hline Ácido benzóico & Toxicidad para peces, algas y bacterias. \\
\hline Ácido fluorhídrico & $\begin{array}{l}\text { Muy corrosivo, reacciona violentamente con muchas sustancias. Es un ácido fuerte, reacciona } \\
\text { violentamente con bases y es corrosivo . Reacciona violentamente con muchos compuestos } \\
\text { originando peligro de fuego y explosión. En contacto con el aire desprende humos corrosivos } \\
\text { más pesados que el aire los cuales se desplazarán a ras del suelo. Ataca al vidrio y a los } \\
\text { compuestos que contengan silíceo. Puede causar hipocalcemia y alteraciones cardiacas y renales. } \\
\text { Se aconseja firmemente impedir que el producto químico se incorpore al ambiente. }\end{array}$ \\
\hline Ácido fosfórico & Puede ser peligrosa para el ambiente; debería prestarse atención especial al agua y el aire. \\
\hline Ácido pícrico & Experimentación en animales muestra que puede causar malformaciones congénitas en humanos \\
\hline Ácido sulfhídrico & Sustancia muy tóxica para organismos acuáticos. \\
\hline Ácido sulfúrico & $\begin{array}{l}\text { Efecto perjudicial en organismos acuáticos por desviación del pH. Tóxico sobre peces y algas. Los } \\
\text { síntomas del edema pulmonar no se ponen de manifiesto, a menudo, hasta pasadas algunas } \\
\text { horas y se agravan por el esfuerzo físico. No verter NUNCA agua sobre esta sustancia; cuando se } \\
\text { deba disolver o diluir, añadirla al agua siempre lentamente. Almacenar en un área con suelo de } \\
\text { hormigón resistente a la corrosión. }\end{array}$ \\
\hline Ácido tartárico & $\begin{array}{l}\text { Efecto perjudicial en organismos acuáticos por desviación del pH. Corrosivo incluso en forma } \\
\text { diluida. }\end{array}$ \\
\hline Aluminio sulfato & Puede ser peligroso para el ambiente; debería prestarse atención especial a los peces. \\
\hline Alfa-naftilamina & $\begin{array}{l}\text { Nociva para organismos acuáticos. Se aconseja firmemente impedir su incorporación al medio } \\
\text { ambiente }\end{array}$ \\
\hline Amonilina dicromato & Sustancia muy tóxica para el medio acuático. Efectos prolongados. Carcinógena. \\
\hline & Puede ser peligrosa para medio ambiente especialmente el acuático. \\
\hline
\end{tabular}

sigue... 


\section{EDUCACIÓN QUÍMICA PARA UN FUTURO SOSTENIBLE}

\begin{tabular}{|c|c|}
\hline Bario cloruro & $\begin{array}{l}\text { Peligro para el agua potable por filtración en suelos y acuíferos. Posible formación de mezclas } \\
\text { nocivas con el agua. }\end{array}$ \\
\hline Bario hidróxido & Peligroso para el agua potable. Efecto perjudicial por desviación del pH. No tirar por el sumidero. \\
\hline Benceno & $\begin{array}{l}\text { Tóxico, muy tóxico en organismos acuáticos, carcinógeno. Por evaporación a temperatura } \\
\text { ambiente puede alcanzar rápidamente concentraciones nocivas. }\end{array}$ \\
\hline Benceno nitro & Tóxico para el medio ambiente. \\
\hline Benzaldehído & Nocivo para organismos acuáticos. \\
\hline Bromo & Tóxico, corrosivo, nocivo para el medio ambiente. \\
\hline Cadmio cloruro & $\begin{array}{l}\text { La sustancia es tóxica para los organismos acuáticos. Bioacumulación, especialmente en plantas. } \\
\text { Se aconseja firmemente impedir que el producto químico se incorpore al ambiente. }\end{array}$ \\
\hline Cadmio óxido & $\begin{array}{l}\text { Bioacumulación, especialmente en plantas y alimentos procedentes del mar. Se aconseja } \\
\text { firmemente impedir que el producto químico se incorpore al ambiente. }\end{array}$ \\
\hline Carbono tetracloruro & $\begin{array}{l}\text { Muy tóxico para ser humano y ambiente, especialmente acuático. Por evaporación a temperatura } \\
\text { ambiente puede alcanzar rápidamente concentraciones nocivas. En corta exposición, la sustancia } \\
\text { irrita los ojos. Puede causar efectos en el sistema nervioso central, dando lugar a una pérdida del } \\
\text { conocimiento. El contacto prolongado o repetido con la piel puede producir dermatitis. } \\
\text { Posiblemente carcinógena para los seres humanos. }\end{array}$ \\
\hline Ciclohexano & Nocivo para organismos acuáticos. \\
\hline Cobalto (II) nitrato & $\begin{array}{l}\text { Los efectos de esta sustancia sobre el medio ambiente no han sido investigados pero los datos } \\
\text { tomados a partir del ion cobalto sugieren que puede ser peligroso para los organismos acuáticos. } \\
\text { Indicado examen médico periódico dependiendo del grado de exposición. Ninguna persona que } \\
\text { haya mostrado síntomas de asma debe entrar nunca en contacto con esta sustancia. }\end{array}$ \\
\hline Cloro & $\begin{array}{l}\text { Esta sustancia puede ser peligrosa para el ambiente; debería prestarse atención especial a las } \\
\text { aves, a los mamíferos y a los organismos acuáticos. }\end{array}$ \\
\hline Cloroformo & $\begin{array}{l}\text { Evitar exposición en niños y adolescentes. Por evaporación a temperatura ambiente puede } \\
\text { alcanzar rápidamente concentraciones nocivas en el aire. Posiblemente carcinógeno. Peligroso } \\
\text { para medio ambiente en especial el acuático. }\end{array}$ \\
\hline Cobalto dicloruro & $\begin{array}{l}\text { Esta sustancia puede ser peligrosa para el ambiente. Debería prestarse atención especial a los } \\
\text { organismos acuáticos. }\end{array}$ \\
\hline Cobre (I) cloruro & $\begin{array}{l}\text { Toxicidad aguda. Muy tóxico para organismos acuáticos. Los iones cobre son tóxicos para peces, } \\
\text { algas, protozoos y bacterias. }\end{array}$ \\
\hline Cobre (II) cloruro & $\begin{array}{l}\text { Muy tóxico para organismos acuáticos. A largo plazo puede provocar efectos negativos en el } \\
\text { medio ambiente acuático. }\end{array}$ \\
\hline Cobre (II) nitrato & $\begin{array}{l}\text { Nocivo por ingestión. Irrita los ojos y la piel. Muy tóxico para los organismos acuáticos, puede } \\
\text { provocar a largo plazo efectos negativos en el medio ambiente acuático. }\end{array}$ \\
\hline Cobre (II) sulfato & $\begin{array}{l}\text { Muy tóxico para organismos acuáticos. Bioacumulación. Se aconseja impedir su incorporación al } \\
\text { medio ambiente. }\end{array}$ \\
\hline Dimetilamina & Muy tóxica para organismos acuáticos. \\
\hline Difenilamina & Muy tóxico para organismos y medio ambiente acuáticos. \\
\hline Etilo acetato & Esta sustancia puede ser peligrosa para el ambiente especialmente el acuático. \\
\hline Fenol & Muy tóxico para ser humano y organismos acuáticos. \\
\hline Formol (formaldehído) & $\begin{array}{l}\text { Tóxico por inhalación, ingestión y contacto. Posible cancerígeno. No incorporar a desagües. } \\
\text { Riesgo de explosión. Tóxico para organismos acuáticos. }\end{array}$ \\
\hline Hierro (III) nitrato & $\begin{array}{l}\text { Toxicidad para los peces. Los nitratos en general pueden favorecer la eutrofia de acuíferos. No } \\
\text { incorporar a suelos ni acuíferos }\end{array}$ \\
\hline Magnesio & $\begin{array}{l}\text { El magnesio quema con llama intensa. Evitar mirar directamente a los fuegos causados por el } \\
\text { magnesio. Reacciona violentamente con agentes extintores de incendio tales como agua, polvo, } \\
\text { dióxido de carbono y halones. Tras reacción, efecto tóxico sobre organismos acuáticos. Efecto } \\
\text { perjudicial por desviación del pH. }\end{array}$ \\
\hline Manganeso (IV) óxido & \\
\hline
\end{tabular}




\section{Mercurio y sus compuestos}

Muy peligrosos, tóxicos. Evitar contacto. El mercurio es muy tóxico para los organismos acuáticos. En la cadena alimentaria referida a los seres humanos tiene lugar bioacumulación, concretamente en los peces y de éstos a los seres humanos. Se aconseja firmemente impedir que el mercurio o los compuestos de mercurio se incorporen al medio ambiente y en especial en el acuático donde pueden causar efectos prolongados a largo plazo.

\begin{tabular}{l} 
Naftaleno \\
\hline Plata nitrato \\
\hline Plomo
\end{tabular}
Tóxico para seres humanos y organismos acuáticos. Puede ser peligroso para medio ambiente, especialmente peces.

Debería prestarse atención especial a los organismos acuáticos. En la cadena alimentaria referida a los seres humanos tiene lugar bioacumulación, concretamente en los organismos acuáticos. Se aconseja firmemente impedir que el producto químico penetre en el ambiente. Los compuestos de plomo en general son tóxicos para el medio ambiente.

Plomo (II) carbonato

Impedir su incorporación al medio ambiente. Tóxico. Bioacumulación. En la cadena alimentaria referida a los seres humanos tiene lugar bioacumulación, concretamente en plantas y mamíferos.

Está indicado examen médico periódico dependiendo del grado de exposición. NO llevar a casa la ropa de trabajo. Se aconseja firmemente impedir que el producto químico se incorpore al ambiente.

\begin{tabular}{|c|c|}
\hline Plomo (II) nitrato & $\begin{array}{l}\text { Esta sustancia puede ser peligrosa para el ambiente. Debería prestarse atención especial a los } \\
\text { organismos acuáticos. En la cadena alimentaria referida a los seres humanos tiene lugar } \\
\text { bioacumulación. Muy tóxico para los organismos acuáticos. Se aconseja firmemente impedir que } \\
\text { el producto químico penetre en el ambiente. }\end{array}$ \\
\hline Plomo (II) yoduro & Tóxico. Bioacumulación. \\
\hline Plomo (IV) óxido & $\begin{array}{l}\text { Alteraciones reproducción humana, nocivo para medio ambiente. Bioacumulación. Muy tóxico } \\
\text { para organismos acuáticos. }\end{array}$ \\
\hline Plomo (VI) cromato & Nocivo para medio ambiente. Carcinógeno. \\
\hline Potasio & Efecto perjudicial por desviación del pH (formación de $\mathrm{KOH}$ ). No incorporar a suelos ni acuíferos. \\
\hline Potasio carbonato & Toxicidad aguda. Efecto perjudicial por desviación del pH \\
\hline Potasio clorato & $\begin{array}{l}\text { Clasificada como agente contaminante del agua en algunos países. Se puede volver inestable } \\
\text { frente al choque si está contaminada con sustancias orgánicas, materiales reductores, polvos } \\
\text { metálicos. NO llevar a casa la ropa de trabajo. Enjuagar la ropa contaminada con agua } \\
\text { abundante (peligro de incendio). }\end{array}$ \\
\hline Potasio cromato & Muy tóxico para organismos acuáticos. \\
\hline Potasio dicromato & Muy tóxico para organismos acuáticos. Carcinógeno. \\
\hline Potasio hidróxido & $\begin{array}{l}\text { Esta sustancia puede ser peligrosa para el ambiente; debería prestarse atención especial a los } \\
\text { organismos acuáticos. }\end{array}$ \\
\hline Potasio nitrato & $\begin{array}{l}\text { Para nitratos en general: pueden favorecer la eutrofia de acuíferos. Peligrosos para el agua } \\
\text { potable. No incorporar a suelos ni acuíferos. }\end{array}$ \\
\hline Potasio permanganato & Muy tóxico para organismos acuáticos. No permitir que se incorpore al ambiente. \\
\hline Sodio & Efecto perjudicial por desviación del pH. No incorporar a suelos ni acuíferos. \\
\hline Sodio acetato & Débilmente acumulativo. No incorporar a suelos ni acuíferos \\
\hline Sodio bisulfito & A pesar de la dilución, forma todavía mezclas tóxicas con el agua. \\
\hline Sodio dicromato & Carcinógeno. Muy tóxico para el medio acuático. Efectos prolongados. \\
\hline Sodio hidróxido & Puede ser peligrosa para el ambiente, especialmente para la vida acuática. \\
\hline Sodio nitrato & $\begin{array}{l}\text { No es de esperar acumulación. Puede favorecer la eutrofia de acuíferos. Peligroso para el agua. } \\
\text { No incorporar a suelos ni acuíferos (general para los nitratos). }\end{array}$ \\
\hline Sodio tiosulfato & Peligroso para el medio ambiente. Evitar su incorporación al agua. \\
\hline Xileno & Puede ser peligroso para el medio ambiente. Atención especial peces y crustáceos. \\
\hline Yodo & Muy tóxico para los organismos acuáticos. No permitir que se incorpore al medio ambiente. \\
\hline Zinc cloruro & $\begin{array}{l}\text { Muy tóxico para los organismos acuáticos. Impedir su incorporación al ambiente. Peligroso para } \\
\text { el agua potable }\end{array}$ \\
\hline Zinc sulfato & $\begin{array}{l}\text { Efecto perjudicial en organismos acuáticos. Puede provocar efectos negativos a largo plazo en el } \\
\text { medio ambiente acuático. No incorporar a suelos ni acuíferos. }\end{array}$ \\
\hline
\end{tabular}

